Rythme. C'est l'effet produit sur l'esprit d'un observateur par le retour régulier de certaines syllabes ou de certains sons importants. Cette importance peut être due à la longueur, au diapason, ou au timbre, c'est ce qu'on nomme 'l'accent' d'une langue, mais il faut se rendre compte que ce qui constitue cet accent dans une langue n'est pas issu de la même combinaison de longueur, diapason et timbre, que dans une autre.

Intonation. Notre habitude $\mathrm{du}$ système des tons dans notre langue maternelle nous persuade qu'elle ne renferme aucun système de ce genre. Mais lorsque nous entendons parler français avec une intonation anglaise nous avons l'impression que cela n'est pas bien. Ce qui peut être obtenu par des changements d'intonations dans une langue ne peut l'être par le même changement dans une autre langue. Suivent des exemples de modifications de tons en Yorouba et en Luganda.

\title{
LA BIBLIOGRAPHIE ETHNOLOGIQUE
}

\section{PAR M. HEYdRICH}

$$
\text { (Voir p. 38I) }
$$

IL n'existe guère jusqu'ici de bibliographies se rapportant aux œuvres pourtant si nombreuses ayant trait à l'ethnologie, et les tentatives faites autrefois pour les réunir ont été abandonnées. On doit mentionner, cependant, des bibliographies partielles et en première ligne celle des Américanistes publiée par le Dr. P. Rivet. Il y a peu de temps encore, l'ethnologue était obligé de consulter pour se documenter les bibliographies d'autres sciences et notamment de la géographie ; quant à celles qui ne renferment que des indications ethnologiques, elles laissent beaucoup à désirer, comme le montre la table des plus importantes parmi celles qui se rapportent à l'Afrique.

C'est pour remédier à cette lacune qu'a été fondé l'Etbnologische Anzeiger, Verlag E. Schweizerbart, Stuttgart, Allemagne. La bibliographie annuelle, classée par région, comprend les publications ethnologiques et archéologiques (ces dernières pour l'Europe seulement) qui ont paru l'année précédente; les plus importants travaux, intéressant les sciences appelées à collaborer, comme l'anthropologie, la linguistique et la géographie humaine, y sont aussi mentionnés. En dehors des cuvres purement scientifiques on y trouvera l'indication d'études pouvant intéresser l'ethnologie, comme celles émanant de missionnaires ou se rapportant aux colonies ou au folklore.

Une partie spéciale formant supplément contiendra la critique des nouveautés importantes. On attirera en particulier l'attention sur les ouvrages parus dans des langues peu courantes comme le russe, le suédois, et le japonais. Enfin, une chronique particulière contiendra des renseignements sur les explorations, les événements scientifiques, les universités, les musées, et les personnalités. Les comptes rendus paraîtront en allemand, en anglais ou en français. 'Tout appui, toute collaboration pour cette œuvre sera bienvenu. 